\title{
New insight into plaque healing after plaque rupture with subsequent thrombus formation detected by intravascular ultrasound
}

A 61 year old man with a three week history of unstable angina was admitted for cardiac catheterisation. Coronary angiography of the right coronary artery (RCA) showed a proximal concentric stenosis and distally a lesion that appeared to be a coronary aneurysm (A and B).

However, intravascular ultrasound (IVUS) identified the lesion as a plaque disruption (D). The arrow indicates the opening of the plaque rupture and the star indicates the false lumen of the washed out plaque. IVUS also showed an adherent thrombus within the cavity (E, arrowhead pointing to the thrombus). In the proximal stenosis IVUS showed a heavily calcified lesion with $>270^{\circ}$ of acoustic shadowing (C).
Plaque disruption and subsequent washing out of the atheromatous content of the core induces thrombus formation and may lead to acute coronary syndromes. Recently, plaque healing was described as another possible result of plaque disruption. Our finding of a thrombus in the emptied cavity of a ruptured plaque may represent the key mechanism in the healing process.

Because of the diffusely diseased RCA and further significant lesions in the left coronary artery the patient had coronary artery bypass graft surgery. He left hospital 10 days after operation and was well six months later.

ALLEN JEREMIAS UNBO GE RAIMUND ERBEL

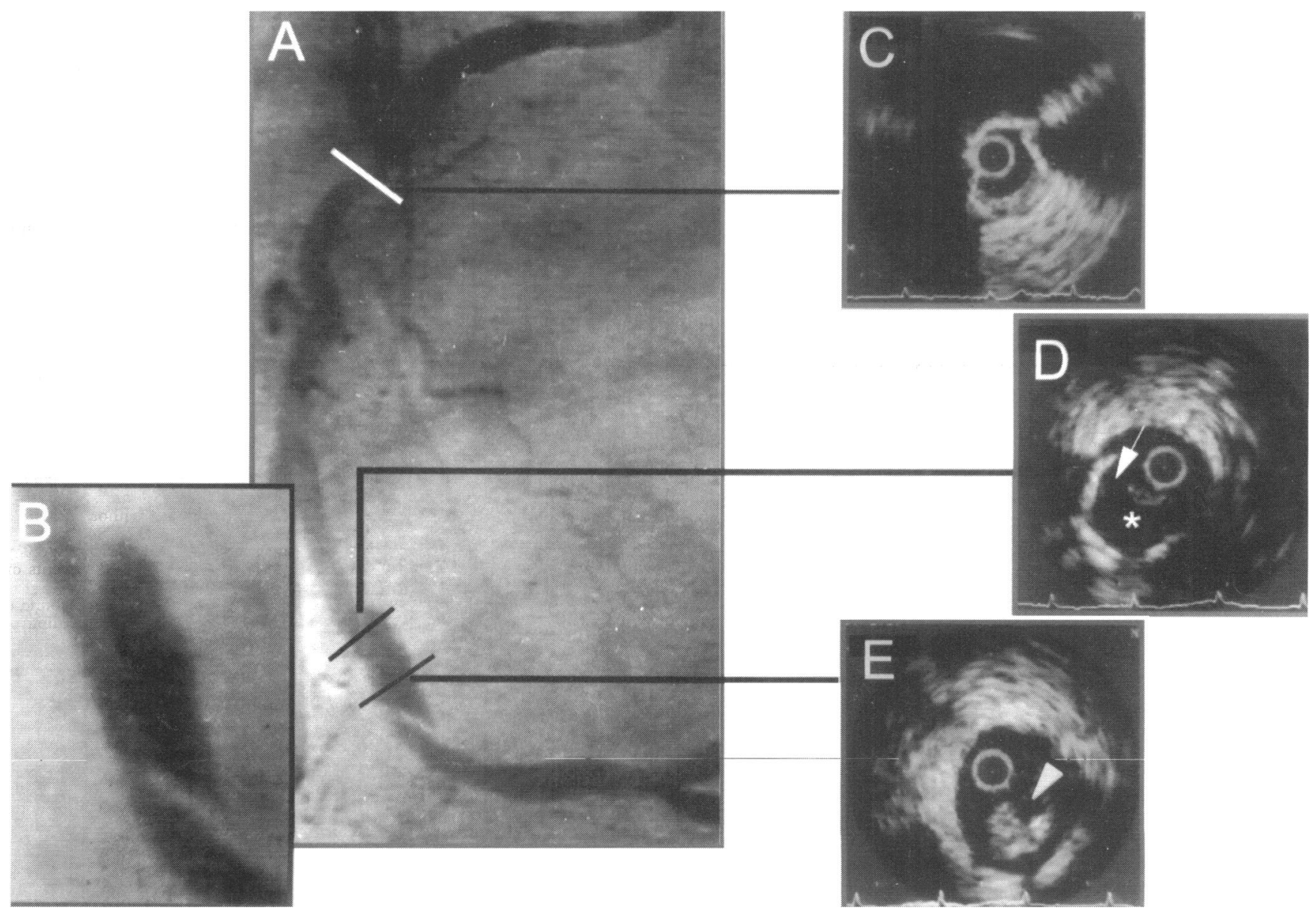

